\title{
Current status and future prospects of the strategy of combining CAR-T with PD-1 blockade for antitumor therapy (Review)
}

\author{
JINJING XU ${ }^{1,2 *}$, QING ZHANG ${ }^{1 *}$, KANG TIAN $^{1}$, HAIYU WANG $^{1}$, HONG YIN $^{1}$ and JUNNIAN ZHENG ${ }^{1,3}$ \\ ${ }^{1}$ Cancer Institute of Xuzhou Medical University, Xuzhou, Jiangsu 221002; ${ }^{2}$ Department of Galactophore, Jiangsu Huai'an Maternity \\ and Children Hospital, Huai'an, Jiangsu 223001; ${ }^{3}$ Jiangsu Center for The Collaboration and Innovation of Cancer Biotherapy, \\ Cancer Institute, Xuzhou Medical University, Xuzhou, Jiangsu 221002, P.R. China
}

Received August 10, 2016; Accepted August 4, 2017

DOI: $10.3892 / \mathrm{mmr} .2017 .8129$

\begin{abstract}
The immune system serves an important role in controlling and eradicating malignant cells. Immunotherapy for treating tumors has received much attention in recent years due to its marked effect. There are two approaches which currently lead this field: Chimeric antigen receptor-modified T-cell immunotherapy (CAR-T) and programmed cell death protein-1 blockade (PD-1 blockade). CAR-T has emerged as a promising regimen for the treatment of a range of types of cancer, including chronic lymphoid leukemia and neuroblastoma, with studies of long term remission in certain patients. PD-1 blockade has been reported to exert marked clinical responses in patients against a range of types of solid cancer, including advanced melanoma, non-small-cell lung cancer and renal cell carcinoma, in addition to hematological malignancies. While increasing the power of the immune system to fight cancer has been a long-standing goal in oncology, a number of studies have demonstrated the synergistic antitumor effects of combination therapies under the umbrella of immunotherapy. The present review focused on a novel combination approach involving CAR-T and PD-1 blockade. The present reviews aimed to discuss the following four aspects of such an approach: i) Current monotherapy status; ii) rationale for the combination of CAR-T and PD-1 blockade; iii) current status of the combination of CAR-T and PD-1 blockade; and iv) conclusions and future perspectives.
\end{abstract}

Correspondence to: Mr. Junnian Zheng, Jiangsu Center for The Collaboration and Innovation of Cancer Biotherapy, Cancer Institute, Xuzhou Medical University, 84 Huaihai West Road, Xuzhou, Jiangsu 221002, P.R. China

E-mail: jnzheng@xzmc.edu.cn

${ }^{*}$ Contributed equally

Key words: chimeric antigen receptor-modified T-cell immunotherapy, programmed cell death protein 1 blockade, combination therapy, immunotherapy, antitumor, efficacy, toxicity

\section{Contents}

1. Introduction

2. Current monotherapy status

3. Rationale for the combination of CAR-T and PD-1 blockade

4. Current status of the combination of CAR-T and PD-1 blockade

5. Conclusions and future perspectives

\section{Introduction}

Chimeric antigen receptor (CAR) T cells, an example of adoptive cellular immunotherapy, and is a potentially curative therapy for a multitude of cancer types (1). CARs are engineered fusion proteins that generally consist of an extracellular single-chain variable fragment ( $\mathrm{scFv}$ ) of an antibody for target recognition, the transmembrane domain that is fused with co-stimulation signaling domains, such as cluster of differentiation (CD) 28 or $4-1 \mathrm{BB}$, and a $\mathrm{CD} 3 \xi$ signaling domain to provide T-cell activation signals (2-4). Additionally, antigen recognition by CARs occurs in a major histocompatibility complex (MHC)-independent manner, in order to overcome the tumor's immune escape by downregulation of $\mathrm{MHC}$ molecules on the cell surface $(5,6)$.

Targeted immunotherapy using chimeric antigen receptor (CAR) molecules to redirect the specificity of cytotoxic T-cells has emerged as a promising strategy for the treatment of a broad range of malignancies $(7,8)$. However, despite encouraging outcomes, accumulating evidence has demonstrated that the immunosuppressive microenvironment induced by tumors and host regulatory cells may limit the full potential of adoptive T-cell immunotherapy (9). Tumors may evade immune surveillance by stimulating immune inhibitory receptors, including hepatitis A virus cellular receptor 2 (TIM-3), cytotoxic T-lymphocyte protein-4 (CTLA-4) and programmed cell death protein-1 (PD-1), on T-cells (10). An example regulatory pathway includes PD-1/programmed cell death protein ligand 1 (PD-L1), which acts as a negative feedback loop to switch off adaptive immunity following the initial immune response (11). The CAR-T and PD-1 blockade techniques have achieved notable results in the therapy numerous types of cancers $(12,13)$. However, a number of clinical trials have 
demonstrated that the efficacy of CAR-T and PD-1 blockade therapy remains limited (14-16). Due to these reported issues, the present review aimed to discuss the status of combination therapies using a combination of CAR-T and PD-1 blockade.

\section{Current monotherapy status}

CAR-T technology, a promising immunotherapy tool, utilizes artificial T-cell surface receptors that stimulate the physiological functions of the native T-cell receptor (TCR) (16). The CAR is composed of an extracellular antigen recognition domain, a spacer, a transmembrane domain and an intracellular T-cell activation domain $(5,17)$. Through the use of genetic modification techniques, effector T-cells may be induced to exhibit improved properties with regard to targeting, killing activity and durability, compared with conventional immunotherapies. CARs combine the effector functions of T lymphocytes with the ability of antibodies to recognize predefined surface antigens with high specificity and avidity, independent of major histocompatibility complex restriction $(18,19)$. Additionally, compared with other T-cell immunotherapy strategies, CAR-T-cells may overcome the local immunosuppressive tumor microenvironment and break down host immune tolerance to tumor cells. CAR-T therapies have generated encouraging results for treating malignant tumors in clinical trials, including cluster of differentiation (CD) 20 for the treatment of non-Hodgkin's lymphoma (20), GD2 for neuroblastoma (21), and CD19 for chronic lymphocytic leukemia (22). However, the application of such therapies to solid tumors has been less encouraging due to a number of factors, including the difficulty in identifying unique tumor-associated antigens, inefficient homing of CAR-T-cells to tumor locations, low persistence of CAR-T-cells following infusion and their functional impairment in the immunosuppressive microenvironment of solid tumors $(23,24)$. Meanwhile, numerous additional potential risks and challenges must be addressed, including the potential for off-target effects, insertion mutations, immune evasion, tumor lysis syndromes and B-cell aplasia.

Tumors are associated with the immune system, and may evade immune surveillance by stimulating immune inhibitory receptors (25). TIM-3, CTLA-4 and PD-1 are all inhibitory receptors with sustained expression in T-cells which may be involved in tumor immune evasion (26). The PD-1/PD-L1 axis, a potential barrier to adoptive T-cell immunotherapeutic strategies, is rapidly emerging as a clinically important immune inhibitory pathway (27). PD-1 is an inhibitory receptor expressed by activated T-cells, activated B cells, natural killer cells and myeloid cells (28). PD-1 inhibits T-cell activation when engaged by its ligands PD-L1 or PD-L2, which are expressed on tumor cells and stromal cells (29). The interaction of PD-L1 with PD-1 may provide an inhibitory signal to induce apoptosis and to suppress the activation or proliferation of T-cells, meaning that immune-checkpoint inhibitors may block the inhibitory signal of T-cells to prevent T-cell anergy (30-32). Previously, checkpoint inhibitor therapies, including PD-1 blockade (Fig. 1), which promote T-cell responses by preventing T-cell exhaustion and anergy, have been reported to exert marked antitumor responses in patients with renal clear cell carcinoma (ccRCC) (33), non-small-cell lung cancer (34), advanced melanoma (35), urothelial carcinoma (36) and other solid tumors $(37,38)$, in addition to lymphoid malignancies (39). Similar to other cancer therapies, toxicity remains a concern. Toxicity associated with PD-1 blockade is typically immune-associated, and may include pneumonitis, colitis, hepatitis, hypophysitis and thyroiditis $(34,40)$.

\section{Rationale for the combination of CAR-T and PD-1 blockade}

A prominent example of a clinically successful CAR-T therapy is the treatment of hematological malignancies using a second-generation CD19-specific CAR, which has demonstrated antitumor activity in clinical trials $(41,42)$. However, the application of CAR-T-cells in the treatment of solid tumors is associated with a number of challenges; one important obstacle is the immunosuppressive effects of tumors (43). The early success of checkpoint inhibitors in enhancing T-cell immunity presented the possibility that these reagents may be used to enhance the antitumor activity of genetically-modified T-cells (44). The most successful cases reported for CAR-T have involved hematological lymphoid malignancies (45), whereas blockade of the PD-1/PD-L1 pathway has demonstrated signs of efficacy against solid tumors $(46,47)$. It was hypothesized that CAR-T in combination with PD-1 blockade may be a promising immunotherapeutic strategy for tumors, which may enhance the antitumor efficacy and extend the scope of treatment.

An improved understanding of the mechanisms of action of CAR-T may aid the design of novel CAR-T-based combination therapies. CAR is an artificial T-cell surface receptor which stimulates the physiological functions of the native TCR (17). Common elements of all CARs include a single-chain antibody for antigen recognition on the surface of tumor cells, and a membrane domain and intracellular signaling domains borrowed from the $\mathrm{CD} 3 \zeta$ chain and costimulatory receptors, including CD28, CD137 and CD27, to supply a costimulatory signal, which appears to be important for expansion and persistence in vivo $(48,49)$.

Additionally, the mechanisms of action for PD-1 blockade merit further investigation. PD-1 is an inhibitory receptor expressed by activated T-cells, activated B cells, natural killer cells and myeloid cells (29). Engagement of the PD-1/PD-L1 pathway results in the phosphorylation of tyrosine-based motifs in the cytoplasmic tail of the PD-1 inhibitory receptor, which promotes the recruitment of tyrosine-protein phosphatase non-receptor type 11 (SHP-1), leading to dephosphorylation of phosphatidylinositol 3-kinase (PI3K). The resulting inhibition of PI3K generates downstream activation of RAC- $\alpha$ serine/threonine protein kinase, decreasing T-cell activation, proliferation and survival (50) (Fig. 2).

In order to further examine the combination strategy, it is necessary to understand in detail the mechanism of action of the combination approach, which is not completely clear at present. However, certain insights may be obtained from previous studies. For instance, John et al (51) observed a significant decrease in the percentage of $\mathrm{Grl}^{+} \mathrm{CD} 11 \mathrm{~b}^{+}$myeloid-derived suppressor cells (MDSCs) in the tumor microenvironment of mice treated with the combination therapy. L-MDSC 


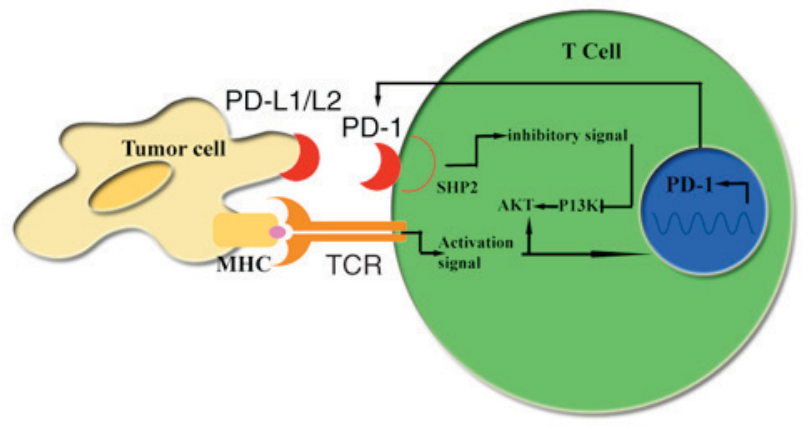

Figure 1. Mechanism of action of PD-1 blockade. PD-1, programmed cell death protein 1; PD-L, PD-2 ligand; MHC, major histocompatibility complex; TCR, T-cell receptor; AKT, RAC- $\alpha$ serine/threonine protein kinase PI3K, phosphatidylinositol 3-kinase; SHP2, tyrosine-protein phosphatase non-receptor type 11 .

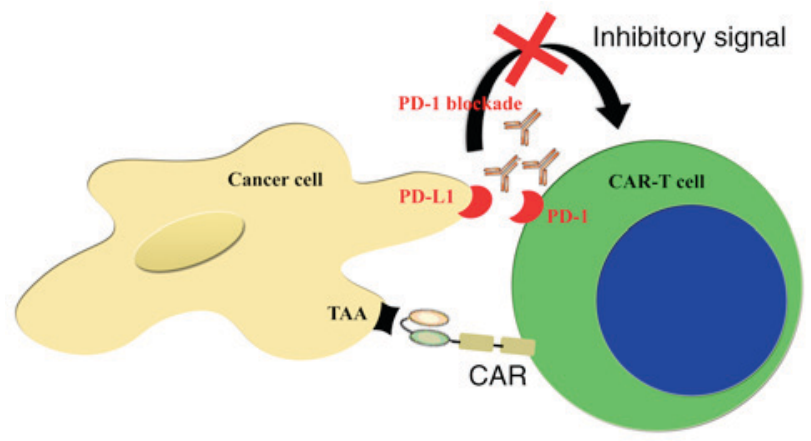

Figure 2. Simplified mechanism of action of the combination of CAR-T and PD-1 blockade. CAR-T, chimeric antigen receptor-modified T-cell immunotherapy; PD-1, programmed cell death protein 1; PD-L1, PD-1 ligand; TAA, tumor-associated antigen.

may circumvent the effects of PD-L1 blockade by exploiting alternative suppressive pathways, including indolamine 2,3-dioxygenase (52), arginase or inducible NO synthase (53). In addition, CAR-T proliferation in the presence of L-MDSC was rescued by SHP-1 and SHP-2 inhibition, which prevented PD-1 signaling within CAR-T (54). The results of ongoing and future studies may facilitate the understanding of the mechanism of action for this combination modality in order to improve patient prognosis.

\section{Current status of the combination of CAR-T and PD-1 blockade}

Immunotherapy frequently utilizes combination approaches to increase efficacy. There are two individual approaches which are currently leading this field: CAR-T and PD-1 blockade (46). Given the promising results from CAR-T and PD-1 blockade monotherapies, it is of importance to investigate whether a combined immunotherapeutic approach involving blockade of the PD-1 pathway may enhance the function of genetically-modified T-cells expressing a CAR, leading to enhanced tumor eradication. Studies into the combination strategy have primarily focused on PD-1 blockade using monoclonal antibody (mAb) and genetic approaches.

An area of interest is CAR-T therapy in combination with PD-1 blockade using mAbs. A study from an Australian group provided promising results; their work demonstrated for the first time that PD-1 blockade was able to enhance the efficacy of CAR-T-cell therapy against established solid tumors in vitro and in vivo (51). The researchers generated primary mouse T-cells expressing an anti-receptor tyrosine-protein kinase erbB-2 (Her-2) CAR containing an extracellular scFv-anti-Her-2 human $\mathrm{mAb}$ region, fused to a transmembrane, intracellular costimulatory CD28 domain and intracellular TCR- $\zeta$ domain. The study examined whether administration of anti-PD-1 monoclonal antibodies was able increase the therapeutic activity of CAR-T-cells against two different Her-2+PD-L1 ${ }^{+}$tumors. Preclinical evidence for the synergistic combination of adoptive T-cell therapy with T-cells expressing CARs and anti-PD-1 mAbs was reported (51). A similar result obtained in a study from Moon et al (55) indicated that the addition of a blocking PD-L1 antibody to an ex vivo CAR tumor infiltrating lymphocyte killing assay was able to restore the defect in tumor cell killing, suggesting that the PD-1 pathway serves a role in maintaining the dysfunction of exhausted CAR-T-cells. An additional similar result reported by Burga et al (56) on the combination of CAR-T and anti-PD-L1 antibodies supported the potential clinical merit of neutralizing L-MDSC in order to allow for optimal antitumor efficacy. The researchers demonstrated that CAR-T therapy in combination with PD-1 blockade through mAbs may be highly synergistic.

A second area of interest is CAR-T therapy in combination with PD-1 blockade through genetic approaches. The results of a previous preclinical trial indicated that anti-carbonic anhydrase IX (CAIX) CAR-T-cells secreting anti-PD-L1 antibodies were able to diminish $\mathrm{T}$-cell exhaustion in vitro and further decrease tumor growth in an orthotopic mouse model of human renal cell carcinoma (RCC). Suarez et al (57) developed a novel CAR therapy for CAIX+RCC that was able to block T-cell exhaustion. The group engineered a bicistronic lentiviral vector to express the anti-CAIX scFv bound to CD28 and $\mathrm{CD} 3 \zeta$ signaling domains in one cassette, and anti-PD-L1 immunoglobulin G1 (IgG1) or IgG4 in a second expression cassette subsequent to an internal ribosome entry site site, thus engineering human anti-CAIX-targeted CAR-T-cells that secreted human anti-PD-L1 antibodies at the tumor site. Compared with the anti-CAIX CAR-T-cells alone in a humanized mouse model of ccRCC, tumor growth was decreased 5-fold and tumor weight was decreased by $50-80 \%$ (57). The results of a preclinical trial performed by Liu et al (43) demonstrated that, while PD-1 blockade augmented the antitumor efficacy of CAR-T-cells, the use of CAR-T-cells expressing PD1CD28 was superior in controlling tumor burden. In order to address this possibility, the researchers used anti-PD1 antibodies in combination with CAR-T-cells, followed by a genetic approach described by others, in which T-cells were transduced with a CAR and a chimeric switch-receptor containing the extracellular domain of PD1 fused to the transmembrane and cytoplasmic domain of the costimulatory molecule CD28. When the PD1 portion of this switch-receptor engages its ligand, PD-L1, it transmits an activating signal via the CD28 cytoplasmic domain instead of the inhibitory signal generally transduced by the PD1 cytoplasmic domain. The aforementioned previous study tested the effect of this PD1CD28 supplement on human CAR-T-cells targeting 
aggressive models of human solid tumors expressing relevant tumor antigens. Treatment of mice bearing large, established solid tumors with PD1CD28 CAR-T-cells led to a significant regression in tumor volume due to enhanced CAR-T-cell infiltration, decreased susceptibility to tumor-induced hypofunction and attenuation of insulin receptor expression, compared with treatment with CAR-T-cells alone or PD-1 antibodies (43). The group demonstrated that CAR-T therapy in combination with PD-1 blockade through genetic approaches may be synergistic.

Combination therapy with CAR-T and PD-1 blockade has been further evaluated in clinical trials. Gargett et al (58) demonstrated that PD-1-targeted combination therapy approaches may be useful for augmenting CAR-T-cell efficacy and persistence in patients. The phase 1 CARPETS trial (registration no. ACTRN12613000198729) utilized GD2-iCAR consisting of CD3ఢ, CD28 and OX40 signaling domains coupled to a $14 \mathrm{~g} 2 \mathrm{a}$ scFv and an inducible caspase- 9 suicide gene, with PD-1 blocked using pembrolizumab. In a protocol amendment for the GRAIN trial of GD2-specific CAR-T-cells in neuroblastoma patients, concurrent treatment with anti-PD-1 mAb was used (58). The researchers applied their understanding of the in vitro results to an analysis of peripheral blood samples derived from patients enrolled in the ongoing CARPETS clinical trial. During the investigations, it was observed that PD-1 blockade restored CAR-T-cell cytokine production and promoted GD2-iCAR T-cell survival and the killing of GD2 ${ }^{+} \mathrm{PD}-\mathrm{L1}^{+}$tumor cells (58). However, the limited number of patients enrolled means that the results that were presented were descriptive and may not be used to form definitive conclusions until more patients are enrolled in the study.

Preclinical studies have illustrated the synergistic efficacy of the combination of CAR-T and PD-1 blockade. By contrast, fewer clinical trials have been performed to evaluate the effects of the combination approach; therefore, whether CAR-T in combination with PD-1 blockade is a rational strategy for clinical trials requires further elucidation. However, it may be hypothesized that if translated to the clinic, PD-1 blockade and CAR-T may be an efficacious treatment, since preclinical evidence supports the synergistic combination of CAR-T and PD-1 blockade.

\section{Conclusions and future perspectives}

The immune system serves an important role in controlling and eradicating malignant cells. Based on the rapid development of immunotherapy, combination therapy using CAR-T and PD-1 blockade has become a novel research area. Preclinical studies have demonstrated that CAR-T and PD-1 blockade are synergistic, leading to long-term survival without causing any signs of pathology in vivo. Moon et al (55) reported that the combination strategy was able to slow tumor growth, although it did not result in regression or cure. Despite recent progress, the field remains at the preclinical phase. However, previous data have suggested that combination therapy may enhance therapeutic efficacy and broaden the range of antitumor treatments $(43,51,55-57,59)$. It may be hypothesized that the combination strategy may be a rational approach for future clinical trials, although further research is required. Prior to wide adoption of the CAR-T and PD-1 blockade combination in clinical practice, a number of challenges must be addressed, including low response rates, toxicity, relatively short response duration, inability to achieve curative effects, and lack of effective and specific tumor-associated antigen targets. Trial-and-error approaches may be used to optimize the strategy in order to provide more rational principles for future clinical practice. At present, further research is required to improve the efficacy and decrease the toxicity of the combination treatment.

Future strategies may improve the efficacy of the combination therapy of CAR-T and PD-1 blockade. Immunotherapy for cancer is primarily dependent on T-cells, particularly $\mathrm{CD}^{+} \mathrm{CTL}$ and $\mathrm{CD}^{+} \mathrm{T}$-helper cells (10). The ability to identify important T-cell characteristics and systematically optimize CAR-T-cell preparation has the potential to markedly improve the efficacy of adoptive T-cell therapy. A previous study demonstrated that CAR-T-cells are enriched in the central memory (TCM) phenotype and that TCM-derived CAR-T-cells are functionally superior to those generated using bulk $\mathrm{CD}^{+}$T-cells (60). Methods to increase the persistence of CAR-T-cells to promote treatment efficacy include using allogeneic virus-specific T-cells and a combination of $\mathrm{CD}^{+}$ TCM cells and CD4+ T-cells $(61,62)$. Strategies to increase the efficacy of CAR-T-cells through the modification of CAR constructs, including the use of 3rd generation and 4th generation armored constructs, are being evaluated (63). An additional approach is to infuse patients with polyspecific CAR-T-cells that target multiple cell surface proteins to prevent immune evasion.

The toxicity of the combined therapy requires further investigation. Due to previous studies of toxicity in certain CAR-T-cell (64), anti-PD-1 (34) and combination CAR-T and PD-1 blockade trials $(51,56,57)$, future studies are required to further optimize the dose and timing regimens of CAR-T-cells with PD-1 blockade in self-antigen mouse models prior to phase I clinical trials. However, identifying an ideal dose of CAR-T-cells to use in combination with PD-1 blockade is difficult as the in vivo expansion of the cells is variable, potentially resulting in inconsistent responses and unpredictable toxicity. Novel methods to increase therapeutic safety are being evaluated and include the introduction of a suicide gene via herpes simplex virus thymidine kinase or inducible caspase- 9 , in addition to the use of targetable cell-surface proteins, including truncated epidermal growth factor receptor or CD20 $(65,66)$. Sadelain et al (5) reported the cotransfection of two different CARs that recognize two different tumor surface antigens, one providing TCR-like signals and the other co-stimulation. The need for simultaneous recognition of two antigens may provide increased specificity and safety.

The combination therapy of CAR-T and PD-1 blockade may be promising for patients with cancer as the research continues and the techniques improve. The combination strategy requires optimization through repeated preclinical and clinical trials in order to minimize toxicity and maximize treatment efficacy for patients with malignancies. The results of ongoing and future studies may facilitate understanding of the differential use of these treatments as a single or a combined modality that improves patient prognosis. It may be hypothesized that immunotherapies will be 
increasingly applied in the clinic due to the rapid development of cellular immunology and molecular biology, and that an era of novel immunotherapies for malignancy may be approaching.

\section{Acknowledgements}

The present study was funded by the National Natural Science Foundation of China (grant no. 81301946), the Natural Science Foundation of Jiangsu Province (grant no. BK2012146) and the Postdoctoral Science Foundation of China (grant nos. 2015T80588 and 2013M540467).

\section{References}

1. Yang JC and Rosenberg SA: Adoptive T-Cell therapy for cancer Adv Immunol 130: 279-294, 2016.

2. Maus MV and June $\mathrm{CH}$ : Making better chimeric antigen receptors for adoptive T-cell therapy. Clin Cancer Res 22: 1875-1884, 2016.

3. Whilding LM and Maher J: CAR T-cell immunotherapy: The path from the by-road to the freeway? Mol Oncol 9: 1994-2018, 2015.

4. Haji-Fatahaliha M, Hosseini M, Akbarian A, Sadreddini S, Jadidi-Niaragh F and Yousefi M: CAR-modified T-cell therapy for cancer: An updated review. Artif Cells Nanomed Biotechnol 44 $1339-1349,2016$.

5. Sadelain M, Brentjens R and Rivière I: The basic principles of chimeric antigen receptor design. Cancer Discov 3: 388-398, 2013.

6. Schreiber RD, Old LJ and Smyth MJ: Cancer immunoediting: Integrating immunity's roles in cancer suppression and promotion. Science 331: 1565-1570, 2011.

7. Lee DW, Kochenderfer JN, Stetler-Stevenson M, Cui YK, Delbrook C, Feldman SA, Fry TJ, Orentas R, Sabatino M, Shah NN, et al: T-cells expressing CD19 chimeric antigen receptors for acute lymphoblastic leukaemia in children and young adults: A phase 1 dose-escalation trial. Lancet 385: 517-528, 2015.

8. Davenport AJ, Jenkins MR, Cross RS, Yong CS, Prince HM, Ritchie DS, Trapani JA, Kershaw MH, Darcy PK and Neeson PJ: CAR-T-cells inflict sequential killing of multiple tumor targeT-cells. Cancer Immunol Res 3: 483-494, 2015.

9. Pardoll DM: The blockade of immune checkpoints in cancer immunotherapy. Nat Rev Cancer 12: 252-264, 2012.

10. Ruella M and Kalos M: Adoptive immunotherapy for cancer. Immunol Rev 257: 14-38, 2014.

11. Keir ME, Butte MJ, Freeman GJ and Sharpe AH: PD-1 and its ligands in tolerance and immunity. Annu Rev Immunol 26 677-704, 2008

12. Riaz IB, Zahid U, Kamal MU, Husnain M, McBride A, Hua A, Hamadani AA, George L, Zeeshan A, Sipra QR, et al: Anti-CD 19 and anti-CD 20 CAR-modified T cells for B-cell malignancies: A systematic review and meta-analysis. Immunotherapy 9 : 979-993, 2017.

13. Taylor A, Rothstein D and Rudd C: Small molecule inhibition of PD-1 transcription is an effective alternative to antibody blockade in cancer therapy. Cancer Res 2017.

14. Sathyanarayanan V and Neelapu SS: Cancer immunotherapy: Strategies for personalization and combinatorial approaches Mol Oncol 9: 2043-2053, 2015.

15. Zhang WL: CAR T-cell therapy: Opportunities and challenges. Immunotherapy 8: 245-247, 2016.

16. Kim MG, Kim D, Suh SK, Park Z, Choi MJ and Oh YK: Current status and regulatory perspective of chimeric antigen receptor-modified T-cell therapeutics. Arch Pharm Res 39: 437-452, 2016

17. Restifo NP, Dudley ME and Rosenberg SA: Adoptive immunotherapy for cancer: Harnessing the T-cell response. Nat Rev Immunol 12: 269-281, 2012.

18. June CH: Principles of adoptive T-cell cancer therapy. J Clin Invest 117: 1204-1212, 2007.

19. June CH: Adoptive T-cell therapy for cancer in the clinic. J Clin Invest 117: 1466-1476, 2007.
20. Till BG, Jensen MC, Wang J, Chen EY, Wood BL, Greisman HA, Qian X, James SE, Raubitschek A, Forman SJ, et al: Adoptive immunotherapy for indolent non-Hodgkin lymphoma and mantle cell lymphoma using genetically modified autologous CD20-specific T-cells. Blood 112: 2261-2271, 2008.

21. Louis CU, Savoldo B, Dotti G, Pule M, Yvon E, Myers GD, Rossig C, Russell HV, Diouf O, Liu E, et al: Antitumor activity and long-term fate of chimeric antigen receptor-positive T-cells in patients with neuroblastoma. Blood 118: 6050-6056, 2011.

22. Brentjens RJ, Riviere I, Park JH, Davila ML, Wang X, Stefanski J, Taylor C, Yeh R, Bartido S, Borquez-Ojeda O, et al: Safety and persistence of adoptively transferred autologous CD19-targeted T-cells in patients with relapsed or chemotherapy refractory B-cell leukemias. Blood 118: 4817-4828, 2011.

23. Kakarla S and Gottschalk S: CAR T-cells for solid tumors: Armed and ready to go? Cancer J 20: 151-155, 2014.

24. Figueroa JA, Reidy A, Mirandola L, Trotter K, Suvorava N, Figueroa A, Konala V, Aulakh A, Littlefield L, Grizzi F, et al: Chimeric antigen receptor engineering: A right step in the evolution of adoptive cellular immunotherapy. Int Rev Immunol 34: 154-187, 2015

25. Bedognetti D, Maccalli C, Bader SB, Marincola FM and Seliger B: Checkpoint inhibitors and their application in breast cancer. Breast care (Basel) 11: 108-115, 2016.

26. Thommen DS, Schreiner J, Muller P, Herzig P, Roller A, Belousov A, Umana P, Pisa P, Klein C, Bacac M, et al: Progression of lung cancer is associated with increased dysfunction of T-cells defined by coexpression of multiple inhibitory receptors. Cancer Immunol Res 3: 1344-1355, 2015.

27. Blank C and Mackensen A: Contribution of the PD-L1/PD-1 pathway to T-cell exhaustion: Anupdateonimplications forchronic infections and tumor evasion. Cancer Immunol Immunother 56: 739-745, 2007.

28. Kedmi M, Avigdor A and Nagler A: Anti-PD-1-targeted therapies focusing on lymphatic malignancies: Biological rationale, clinical challenges and opportunities. Acta Haematol 133: 129-135, 2015.

29. Keir ME, Butte MJ, Freeman GJ and Sharpel AH: PD-1 and its ligands in tolerance and immunity. Annu Rev Immunol 26: 677-704, 2008

30. Yao S and Chen L: PD-1 as an immune modulatory receptor. Cancer J 20: 262-264, 2014.

31. Chen DS, Irving BA and Hodi FS: Molecular pathways: Next-generation immunotherapy-inhibiting programmed death-ligand 1 and programmed death-1. Clin Cancer Res 18: 6580-6587, 2012

32. Okazaki T, Chikuma S, Iwai Y, Fagarasan S and Honjo T: A rheostat for immune responses: The unique properties of PD-1 and their advantages for clinical application. Nat Immunol 14: 1212-1218, 2013.

33. Massari F, Santoni M, Ciccarese C, Santini D, Alfieri S, Martignoni G, Brunelli M, Piva F, Berardi R, Montironi R, et al: PD-1 blockade therapy in renal cell carcinoma: Current studies and future promises. Cancer Treat Rev 41: 114-121, 2015.

34. Topalian SL, Hodi FS, Brahmer JR, Gettinger SN, Smith DC, McDermott DF, Powderly JD, Carvajal RD, Sosman JA, Atkins MB, et al: Safety, activity and immune correlates of anti-PD-1 antibody in cancer. N Engl J Med 366: 2443-2454, 2012.

35. Brahmer JR, Tykodi SS, Chow LQ, Hwu WJ, Topalian SL, Hwu P, Drake CG, Camacho LH, Kauh J, Odunsi K, et al: Safety and activity of anti-PD-L1 antibody in patients with advanced cancer. N Engl J Med 366: 2455-2465, 2012.

36. Ratta R, Zappasodi R, Raggi D, Grassi P, Verzoni E, Necchi A, Di Nicola M, Salvioni R, de Braud F and Procopio G: Immunotherapy advances in uro-genital malignancies. Crit Rev Oncol Hematol 105: 52-64, 2016.

37. Bracarda S, Altavilla A, Hamzaj A, Sisani M, Marrocolo F, Del Buono S and Danielli R: Immunologic checkpoints blockade in renal cell, prostate and urothelial malignancies. Semin Oncol 42: 495-505, 2015.

38. Joshi M, Pal SK and Drabick JJ: Novel approaches in cancer immunotherapy-a light at the end of the tunnel. Discov Med 21: 479-487, 2016

39. Thanarajasingam G, Thanarajasingam U and Ansell SM: Immune checkpoint blockade in lymphoid malignancies. FEBS J 283: 2233-2244, 2016.

40. Weber JS: Practical management of immune-related adverse events from immune checkpoint protein antibodies for the oncologist. Am Soc Clin Oncol Educ Book: 174-177, 2012. 
41. Zhu Y, Tan Y, Ou R, Zhong Q, Zheng L, Du Y, Zhang Q and Huang J: Anti-CD19 chimeric antigen receptor-modified T cells for B-cell malignancies: A systematic review of efficacy and safety in clinical trials. Eur J Haematol 96: 389-396, 2016.

42. Srivastava S and Riddell SR: Engineering CAR-T-cells: Design concepts. Trends Immunol 36: 494-502, 2015.

43. Liu X, Ranganathan R, Jiang S, Fang C, Sun J, Kim S, Newick K, Lo A, June CH,Zhao Y and Moon EK: A chimeric switch-receptor targeting PD1 augments the efficacy of second-generation car T-cells in advanced solid tumors. Cancer Res 76: 1578-1590, 2016.

44. Adachi K and Tamada K: Immune checkpoint blockade opens an avenue of cancer immunotherapy with a potent clinical efficacy. Cancer Sci 106: 945-950, 2015.

45. Chicaybam L, Sodré AL and Bonamino M: Chimeric antigen receptors in cancer immuno-gene therapy: Current status and future directions. Int Rev Immunol 30: 294-311, 2011.

46. Morales-Kastresana A, Labiano S, Quetglas JI and Melero I: Better performance of CARs deprived of the PD-1 brake. Clin Cancer Res 19: 5546-5548, 2013.

47. Postow MA, Callahan MK and Wolchok JD: Immune checkpoint blockade in cancer therapy. J Clin Oncol 33: 1974-1982, 2015.

48. Shirasu N and Kuroki M: Functional design of chimeric T-Cell antigen receptors for adoptive immunotherapy of cancer: Architecture and outcomes. Anticancer Res 32: 2377-2383, 2012.

49. Cheadle EJ, Gornall H, Baldan V, Hanson V, Hawkins RE and Gilham DE: CAR T-cells: Driving the road from the laboratory to the clinic. Immunol Rev 257: 91-106, 2014

50. Blank C, Gajewski TF and Mackensen A: Interaction of PD-L1 on tumor cells with PD-1 on tumor-specific T-cells as a mechanism of immune evasion: Implications for tumor immunotherapy. Cancer Immunol Immunother 54: 307-314, 2005.

51. John LB, Devaud C, Duong CP, Yong CS, Beavis PA, Haynes NM, Chow MT, Smyth MJ, Kershaw MH and Darcy PK: Anti-PD-1 antibody therapy potently enhances the eradication of established tumors by gene-modified T-cells. Clin Cancer Res 19: 5636-5646, 2013.

52. Yu J, Wang Y, Yan F, Li H and Ren X: Response to comment on 'Myeloid-derived suppressor cells suppress antitumor immune responses through IDO expression and correlate with lymph node metastasis in patients with breast cancer'. J Immunol 190: 5341-5342, 2013.

53. Mazzoni A, Bronte V, Visintin A, Spitzer JH, Apolloni E, Serafini P, Zanovello P and Segal DM: Myeloid suppressor lines inhibit T-cell responses by an NO-dependent mechanism. J Immunol 168: 689-695, 2002.

54. Mauldin IS, Tung KS and Lorenz UM: The tyrosine phosphatase SHP-1 dampens murine Th17 development. Blood 119: 4419-4429, 2012.
55. Moon EK, Wang LC, Dolfi DV, Wilson CB, Ranganathan R, Sun J, Kapoor V, Scholler J, Puré E, Milone MC, et al: Multifactorial T-cell hypofunction that is reversible can limit the efficacy of chimeric antigen receptor-transduced human T-cells in solid tumors. Clin Cancer Res 20: 4262-4273, 2014.

56. Burga RA, Thorn M, Point GR, Guha P, Nguyen CT, Licata LA, DeMatteo RP, Ayala A, Joseph Espat N, Junghans RP and Katz SC: Liver myeloid-derived suppressor cells expand in response to liver metastases in mice and inhibit the anti-tumor efficacy of anti-CEA CAR-T. Cancer Immunol Immunother 64: 817-829, 2015.

57. Suarez ER, Chang de K, Sun J, Sui J, Freeman GJ, Signoretti S, Zhu Q and Marasco WA: Chimeric antigen receptor T-cells secreting anti-PD-L1 antibodies more effectively regress renal cell carcinoma in a humanized mouse model. Oncotarget 7 : 34341-34355, 2016.

58. Gargett T, Yu W, Dotti G, Yvon ES, Christo SN, Hayball JD, Lewis ID, Brenner MK and Brown MP: GD2-specific CAR T-cells undergo potent activation and deletion following antigen encounter but can be protected from activation-induced cell death by PD-1 blockade. Mol Ther 24: 1135-1149, 2016.

59. Prosser ME, Brown CE, Shami AF, Forman SJ and Jensen MC: Tumor PD-L1 co-stimulates primary human CD8(+) cytotoxic T-cells modified to express a PD1: CD28 chimeric receptor. Mol Immunol 51: 263-272,2012.

60. Chang ZL, Silver PA and Chen YY: Identification and selective expansion of functionally superior T-cells expressing chimeric antigen receptors. J Transl Med 13: 161, 2015.

61. Terakura S, Yamamoto TN, Gardner RA, Turtle CJ, Jensen MC and Riddell SR: Generation of CD19-chimeric antigen receptor modified CD8+ T-cells derived from virus-specific central memory T-cells. Blood 119: 72-82, 2012.

62. Cruz CR, Micklethwaite KP, Savoldo B, Ramos CA, Lam S, Ku S, Diouf O, Liu E, Barrett AJ, Ito S, et al: Infusion of donor-derived CD19-redirected virus-specific T-cells for B-cell malignancies relapsed after allogeneic stem cell transplant: A phase 1 study. Blood 122: 2965-2973, 2013.

63. Pegram HJ, Park JH and Brentjens RJ: CD28z CARs and Armored CARs. Cancer J 20: 127-133, 2014.

64. Shi H, Liu L and Wang Z: Improving the efficacy and safety of engineered T-cell therapy for cancer. Cancer Lett 328: 191-197, 2013.

65. Jones BS, Lamb LS, Goldman F and Di Stasi A: Improving the safety of cell therapy products by suicide gene transfer. Front Pharmacol 5: 254, 2014.

66. Wang X, Chang WC, Wong CW, Colcher D, Sherman M, Ostberg JR, Forman SJ, Riddell SR and Jensen MC: A transgene-encoded cell surface polypeptide for selection, in vivo tracking, and ablation of engineered cells. Blood 118: 1255-1263, 2011. 